\title{
Additional MRI for initial M-staging in pancreatic cancer: a cost-effectiveness analysis
}

\author{
Felix G. Gassert ${ }^{1}$ (D) Sebastian Ziegelmayer ${ }^{1}$. Johanna Luitjens ${ }^{2}$. Florian T. Gassert ${ }^{1} \cdot$ Fabian Tollens $^{3}$. Johann Rink ${ }^{3}$. \\ Marcus R. Makowski ${ }^{1}$. Johannes Rübenthaler ${ }^{2} \cdot$ Matthias F. Froelich $^{3}$
}

Received: 21 May 2021 / Revised: 21 September 2021 / Accepted: 24 September 2021 / Published online: 27 November 2021

(c) The Author(s) 2021

\begin{abstract}
Objective Pancreatic cancer is portrayed to become the second leading cause of cancer-related death within the next years. Potentially complicating surgical resection emphasizes the importance of an accurate TNM classification. In particular, the failure to detect features for non-resectability has profound consequences on patient outcomes and economic costs due to incorrect indication for resection. In the detection of liver metastases, contrast-enhanced MRI showed high sensitivity and specificity; however, the cost-effectiveness compared to the standard of care imaging remains unclear. The aim of this study was to analyze whether additional MRI of the liver is a cost-effective approach compared to routinely acquired contrastenhanced computed tomography (CE-CT) in the initial staging of pancreatic cancer.

Methods A decision model based on Markov simulation was developed to estimate the quality-adjusted life-years (QALYs) and lifetime costs of the diagnostic modalities. Model input parameters were assessed based on evidence from recent literature. The willingness-to-pay (WTP) was set to $\$ 100,000 / \mathrm{QALY}$. To evaluate model uncertainty, deterministic and probabilistic sensitivity analyses were performed.

Results In the base-case analysis, the model yielded a total cost of $\$ 185,597$ and an effectiveness of 2.347 QALYs for CE-MR/CT and $\$ 187,601$ and 2.337 QALYs for CE-CT respectively. With a net monetary benefit (NMB) of $\$ 49,133$, CE-MR/CT is shown to be dominant over CE-CT with a NMB of $\$ 46,117$. Deterministic and probabilistic survival analysis showed model robustness for varying input parameters.

Conclusion Based on our results, combined CE-MR/CT can be regarded as a cost-effective imaging strategy for the staging of pancreatic cancer.

Key Points

- Additional MRI of the liver for initial staging of pancreatic cancer results in lower total costs and higher effectiveness.

- The economic model showed high robustness for varying input parameters.
\end{abstract}

Keywords Pancreatic neoplasms $\cdot$ Cost-effectiveness $\cdot$ Cancer staging $\cdot$ Magnetic resonance imaging $\cdot$ Multidetector computed tomography

Felix G. Gassert and Sebastian Ziegelmayer contributed equally to this work

Felix G. Gassert

felix.gassert@tum.de

1 Department of Diagnostic and Interventional Radiology, Klinikum Rechts Der Isar, Technical University of Munich, Ismaninger Str. 22, 81675 München, Germany

2 Department of Radiology, University Hospital, LMU Munich, Marchioninistr. 15, 81377 Munich, Germany

3 Department of Radiology and Nuclear Medicine, University Medical Centre Mannheim, Theodor-Kutzer-Ufer 1-3, 68167 Mannheim, Germany

\section{Abbreviations}

CE-CT Contrast-enhanced computed tomography

ICER Incremental cost-effectiveness ratio

NMB Net monetary benefit

QALY Quality-adjusted life year

QOL Quality of life

SCI Standard of care imaging

WTP Willingness-to-pay 


\section{Introduction}

Pancreatic cancer is an exceptionally aggressive tumor entity with the lowest 5-year survival rate of all solid tumors [1]. In addition to pronounced heterogeneity, poor prognosis can be attributed primarily to delayed diagnosis, such that $50 \%$ of pancreatic cancers are already metastatic at initial diagnosis [2]. Due to the increasing incidence, it is predicted that pancreatic cancer will become the second leading cause of cancer-related death in the USA by 2030 , therefore posing a relevant burden to the healthcare systems [3]. Surgical resection usually in terms of pancreaticoduodenectomy followed by adjuvant chemotherapy is the only curative therapeutic option. Despite steady improvements in surgical technique and perioperative management, resection of pancreatic cancer remains a demanding procedure with a postoperative mortality rate of $3-5 \%[4,5]$. In the metastatic stage, patients receive palliative chemotherapy with gemcitabine/nab-paclitaxel or FOLFIRINOX. In particular, FOLFIRINOX was shown to significantly prolong survival, however, at the expense of increased toxicity $[6,7]$. The potential adverse effects of the therapeutic options emphasize the relevance of correct TNM classification, especially with regard to the presence of metastasis, which is a contraindication for surgical resection. For M-staging, the liver as the most frequent site of metastasis is of particular importance [8]. Staging of pancreatic cancer involves biphasic computed tomography of the chest, abdomen, and pelvis to evaluate resectability and rule out metastasis. The detection rate of liver metastases in computed tomography is described in the literature with a sensitivity of 70 to $76 \%[9,10]$. Contrast-enhanced MRI is frequently described as an alternative for assessing the locoregional extent and detecting lymph node and liver metastases. It appears to be dominant over contrastenhanced computed tomography (CE-CT) in detecting liver lesions, with a sensitivity of 90 to $97 \%$ [11, 12]. Furthermore, additional MR imaging during the staging of pancreatic cancer was shown to reduce resection rates, indicating that patients in a metastatic stage who received staging with CE-CT were resected incorrectly [13].

In this context, additional imaging is often deemed expensive. A cost-effectiveness analysis is a tool to assess the impact of potential changes in patient management and its impact on long-term costs and effectiveness. Despite improvement in diagnosis and treatment of pancreatic cancer, no study has been performed to evaluate the utilization of combined contrast-enhanced MRI and CT compared to standard imaging (contrast-enhanced CT) in the detection of features for non-resectability from an economic point of view. Therefore, the aim of our study was to determine the cost-effectiveness of combined CE-MRT/CT in detecting liver metastasis at the initial staging of pancreatic cancer compared to the standard of care imaging (SCI) using CE-CT.

\section{Methods}

\section{Model structure}

A decision model based on Markov simulations was developed using dedicated analysis software (TreeAge Pro Version 19.1.1) to evaluate the cost-effectiveness of each imaging strategy. For the simulation, the Markov model included the following states:

- Alive, non-resectable: i.e., describing patients with initially metastatic disease or non-resectable primary tumor, therefore not undergoing surgery

- Alive, resected, no metastasis or local recurrence: i.e., describing patients after resection without the presence of metastasis or local recurrence

- Alive, resected, presence of R1-situation or local recurrence: i.e., describing patients without metastasis but local recurrence or R1-situation after resection

- Alive, resected, presence of metastasis (with and without local recurrence): i.e., describing patients with presence of metastasis, either associated with recurrence or due to missed metastatic disease on pre-surgical imaging

- Dead

\section{Input parameters}

Ethics approval was not necessary for this retrospective analysis based on commonly available data. The model input parameters were estimated based on evidence from published literature. Age-specific risk of death was derived from the US life tables [14]. The probability to correctly classify tumors as resectable using CE-CT was set to $92.25 \%$; consequently, the false positive rate was 7.75\% [13]. The cost of pancreatic resection with respect to potential complications and differing patient characteristics was set to $\$ 42,869$ [15], which poses a reasonable estimate between $\$ 22,000$ stated by Sutton et. al and $\$ 55,538-\$ 61,806$ by Tramontano et. al $[16,17]$. All input parameters and corresponding references are listed in Table 1.

The Willingness-to-pay (WTP) was set to $\$ 100,000$ per quality-adjusted life year (QALY) at a discount rate of $3 \%$ $[26,27]$.

\section{Costs and utilities}

Starting from the United States (US) healthcare perspective, diagnostic procedure costs were estimated based on 
Table 1 Model input parameters

\begin{tabular}{|c|c|c|}
\hline Variable & Estimate & Source \\
\hline Expected age at diagnostic procedure & 70 years & {$[18]$} \\
\hline Assumed willingness-to-pay per QALY & $\$ 100,000$ & Assumption \\
\hline Discount rate & $3 \%$ & Assumption \\
\hline Markov model time horizon & 5 years & Assumption \\
\hline \multicolumn{3}{|l|}{ Diagnostic test performances } \\
\hline $\mathrm{CT}$ probability of $\mathrm{TP}$ & $92.25 \%$ & [13] \\
\hline $\mathrm{CT}$ probability of $\mathrm{FP}$ & $7.75 \%$ & {$[13]$} \\
\hline \multicolumn{3}{|l|}{ Costs (acute) } \\
\hline CT chest, abdomen, pelvis & $\$ 692$ & Medicare (Ref.No.: 71260+74,177) \\
\hline MRI abdomen & $\$ 615$ & Medicare (Ref.No.: 74183) [19] \\
\hline Surgery & $\$ 42,869$ & {$[15]$} \\
\hline \multicolumn{3}{|l|}{ Costs (long term) } \\
\hline Therapy for patients with M1 & $\$ 60,000$ & {$[17,20]$} \\
\hline Therapy/follow-up after surgery & $\begin{array}{l}\$ 36,126 \text { (first year); } \\
\$ 1,126 \text { (following years) }\end{array}$ & Adapted from [17] \\
\hline Therapy after resection with M1 & $\$ 60,000$ & {$[17,20]$} \\
\hline Therapy with local recurrence / R1 & $\$ 30,000$ & {$[17]$} \\
\hline \multicolumn{3}{|l|}{ Utilities } \\
\hline M1 after surgery & 0.6 & {$[21,22]$} \\
\hline M1 without surgery & 0.65 & [23] \\
\hline M0 post surgery & $\begin{array}{l}0.79 \text { (first year), } \\
0.87 \text { (following years) }\end{array}$ & Adapted from $[21,23]$ \\
\hline Death & 0 & Assumption \\
\hline \multicolumn{3}{|l|}{ Transition probabilities } \\
\hline Proportion of R1-resections & $80 \%$ & [24] \\
\hline Occurrence of metastasis after resection & $38.00 \%$ & {$[25]$} \\
\hline Mortality rate of surgery & $3.70 \%$ & [4] \\
\hline Mortality rate with M1 cancer & $50.74 \%$ & {$[18]$} \\
\hline Probability of death M0 cancer & $2.90 \%$ & [14] \\
\hline
\end{tabular}

Medicare data and available literature (Table 1). Annual costs for patients with respect to different therapy regimens and tumor states were derived from recent literature [17, 20].

Utility was measured in the quality-adjusted life years (QALY) in follow-up after each diagnostic strategy. According to previous studies, quality of life (QOL) for resected patients without metastasis was set to 0.726 in the first year and 0.797 for the following years [21, 22]. For patients with metastasis and with or without surgery, QOL was set to 0.65 and 0.6 respectively [23].

\section{Transition probabilities}

Transition probabilities were derived from a systematic review of the recent literature (Table 1). The perioperative mortality rate within 90 days of pancreatic resection was set to $3.7 \%$ [4]. The probability of secondary occurrence of metastases after resection of the primary tumor was estimated to be $38 \%$ per year [25]. The annual mortality rate of patients with and without metastasis was set to $50.74 \%$ and $2.9 \%$ respectively $[14,18]$.

\section{Cost-effectiveness analysis}

The cost-effectiveness analysis was simulated for Markov run time of 5 years after the initial staging of pancreatic cancer. QALY and costs were calculated for the base case scenario with respect to WTP and discount rate.

To evaluate model uncertainty, deterministic and probabilistic sensitivity analysis was conducted. The deterministic sensitivity analysis was performed by altering the input parameters and observing their influence on the incremental effectiveness, incremental cost, and incremental cost-effectiveness ratio (ICER).

The Monte Carlo modeling was used for probabilistic sensitivity analysis. A total of 30,000 iterations were performed 
to estimate acceptability curves. Furthermore, the net monetary benefit (NMB) with respect to the probability of possible tumor resection was calculated for both imaging strategies.

\section{Results}

\section{Cost-effectiveness analysis}

The decision model and the respective schematic architecture of the Markov model with the potential states of disease are shown in Fig. 1. For the base case scenario with a WTP of \$ 100,000 per QALY and a 5-year time span, the model yielded a total cost and effectiveness of $\$ 187,601$ and 2.337 QALYs for the SCI (CE-CT), whereas combined CE-MR/CT was estimated to cost $\$ 185,597$ with an effectiveness of 2.347 QALYs. The calculated Incremental cost-effectiveness ratio for combined CE-MR/CT was negative, indicating higher effectiveness of CE-MRI/CT at lower costs, i.e., the dominance of this strategy over the alternative. The NMB for CE-MR/CT was $\$ 49,133$ and $\$ 46,117$ for CE-CT.

\section{Probabilistic sensitivity analysis}

At the WTP threshold of $\$ 100,000$ per QALY combined CE-MR/CT remained the cost-effective alternative to CE-CT in the majority of all iterations. Even for a hypothetical reduction in WTP thresholds to $\$ 0$, combined CE-MR/CT remained the costeffective alternative in the vaster majority of iterations. Exemplary iterations of the model with the corresponding costs and effectiveness of the respective modalities are shown in Fig. 2.

\section{Deterministic sensitivity analysis}

A deterministic sensitivity analysis was performed to account for possible variance in induced costs as well as different probabilities of state transition. The incremental cost-effectiveness ratio remained below the WTP threshold for the applied changes in the above-mentioned parameters, indicating the cost-effectiveness of combined CE-MR/CT in the detection of features for non-resectability for the most common variants of the respective parameters. A tornado diagram displaying the changes in ICER is shown for each parameter in Fig. 3. A dedicated one-way sensitivity analysis was performed, to investigate the influence of the proportion of patients resectable. For the broad majority of inputs, i.e., levels below 98.88\%, CT + MRI yielded the higher net monetary benefit when compared to CT alone (Fig. 4).

\section{Discussion}

Due to the increasing incidence combined with extensive and cost-intensive treatment options, the economic burden of pancreatic cancer on the healthcare system is steadily increasing. The detection of liver metastases is of particular importance as they are the most frequent metastases and an exclusion criterion for surgical resection and its associated costs and complications. In the present study, we show that CE-MR/CT is a cost-effective diagnostic strategy in staging pancreatic cancer. The superiority of MRI compared to CT in the diagnosis and staging of pancreatic cancer has already been demonstrated $[28,29]$. However, despite the fact that cost-effectiveness is of increasing importance in health care, studies evaluating the economic implications of various imaging modalities in pancreatic cancer remain sparse. Pamela et al. were able to show that CE-CT, followed by laparoscopy and laparoscopic ultrasonography, was a costeffective diagnostic workup in determining resectability of pancreatic cancer with MRI imaging coming second [30]. However, this analysis was based on lower sensitivity for MRI, potentially due to the state of scanner development at that time. Diffusion-weighted imaging and hepatocytespecific contrast agent were able to greatly increase the sensitivity of MRI in liver lesion detection [31, 32]. Heinrich et al. postulated that ${ }^{18} \mathrm{~F}$ FDG PET-CT is the cost-effective method over CE-CT for assessing the resectability of pancreatic cancer [33]. Whether this method is also dominant over CE-MRI was not evaluated. In our study, we did not integrate ${ }^{18} \mathrm{~F}$ FDG PET-CT as an additional imaging strategy due to the higher sensitivity and specificity at lower cost of CE-MRI reported in the literature. Furthermore, the costeffectiveness of CE-MR in the detection of liver metastases in comparison to different imaging modalities has already been demonstrated for other tumor entities [34].

In our study, combined CE-MR/CT was the dominant strategy compared to CE-CT for a range of WTP-thresholds. Consequently, it would be the dominant diagnostic workup even for lower WTP-thresholds, as in the UK health care system, for instance [35].

Deterministic sensitivity analysis showed a consistent negative ICER for CE-MR/CT for different variations in the input parameters, indicating lower costs at higher effectiveness. In addition, robustness is emphasized by a positive NMB for CE-MR/CT under varying resection probabilities. Despite promising results, the following limitations of our study have to be addressed. It must be emphasized that cost-effectiveness analysis with decision-based models is highly dependent on the input parameters used, and thus an optimal decision for each individual case is not achievable due to deviating parameters. The Markov model used did not differentiate between tumor extent, as this would exceed the scope of the study. Due to the correlation between tumor stage and present metastases, the cost-effectiveness of imaging modalities with respect to tumor stage should be investigated. Moreover, our decision model did not show an extra group for patients with undetected metastases in MRI in the Markov model as input values would be unlikely to be available in the literature. False-negative 


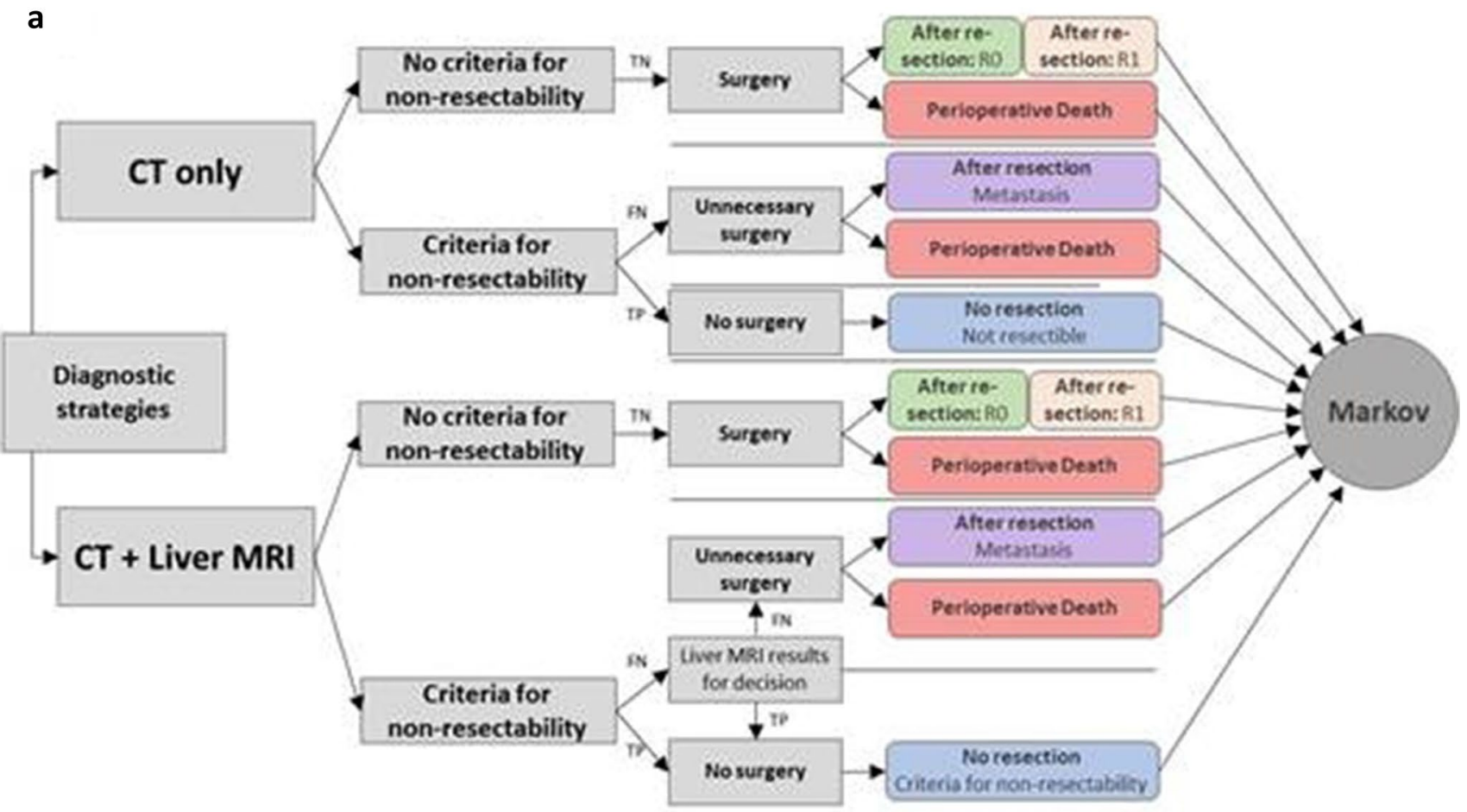

b

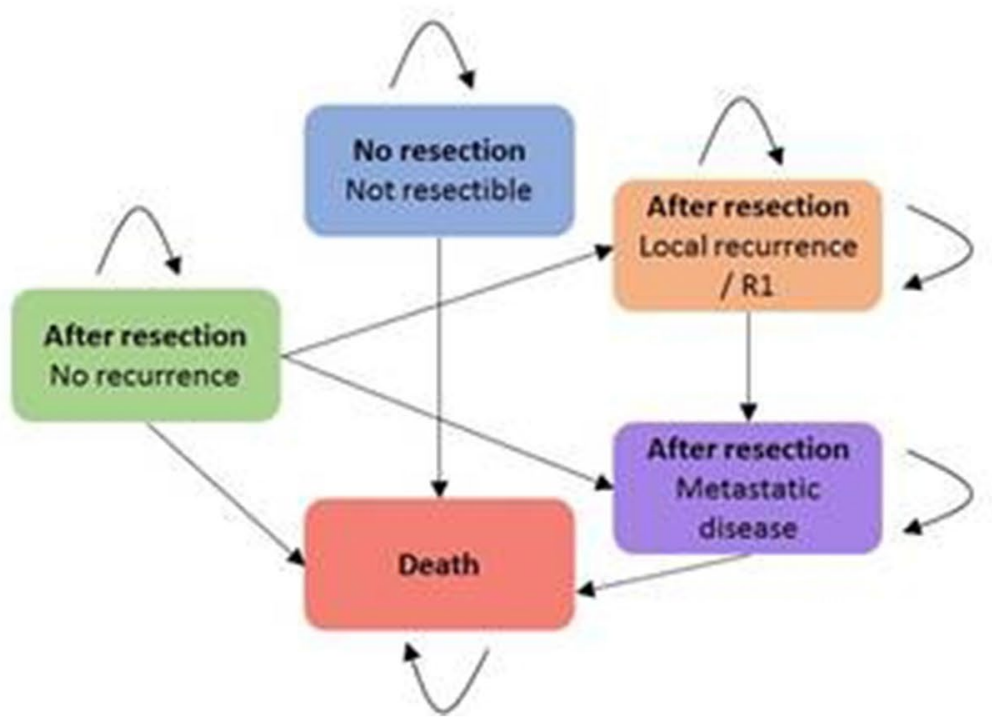

Fig. 1 a Effective alternative to CE-CT schematic overview of the decision model for both diagnostic strategies (CE-CT and CE-MR/ CT). Markov model analysis was conducted for each outcome. $\mathbf{b}$ The Markov model with the respective states and their potential transi-

diagnosis in MRI therefore is reflected through the rate of recurrence after resection of these patients. Functional imaging modalities as ${ }^{18} \mathrm{~F}$ FDG PET-CT or PET-MRI were excluded from the study due to the lack of establishment in clinical practice. In the following studies, these modalities should be tion. The initial state was determined by the outcome in the decision model. TP, true positive; TN, true negative; FP, false positive; FN, false negative, CT, computed tomography; MRI, magnetic resonance imaging

investigated in more detail based on promising results from recent findings [36, 37]. Lastly, this study was performed based on guidelines for the implementation of cost-effectiveness analyses and therefore analyses costs and effectiveness for an average of patients. As a matter of course, not every individual 


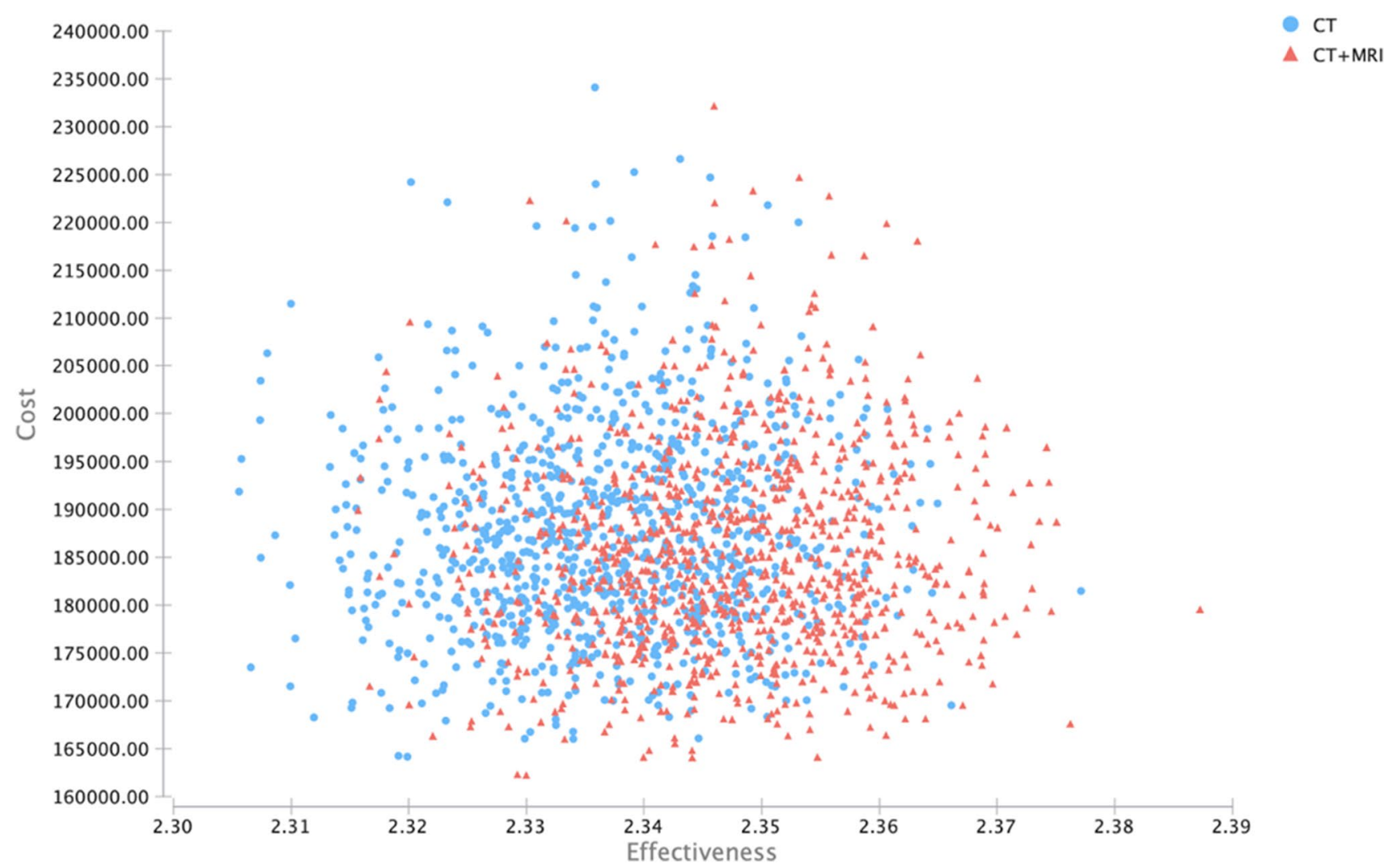

Fig. 2 Scatterplot of cost and effectiveness of CE-MR/CT and CE-CT for exemplary iterations. CT, computed tomography; MRI, magnetic resonance imaging

Fig. 3 Results of the deterministic sensitivity analysis visualized as a tornado diagram, showing the influence of input parameter variation on the incremental cost-effectiveness ratio (ICER). MRI, magnetic resonance imaging; EV, expected value at base case scenario; M1, metastasized
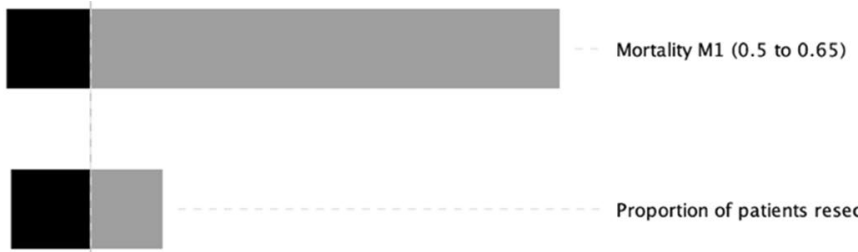

Proportion of patients resectable $(0.8$ to 0.95$)$

Cost of MRI (300 to 759)

Yearly cost of M1-treatment (35000 to 80000 )

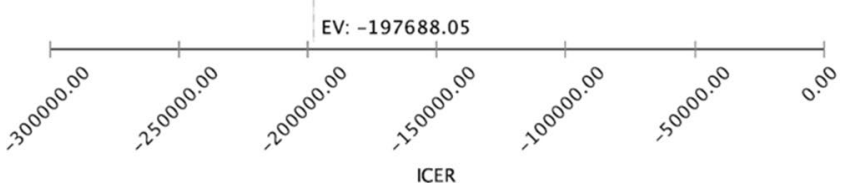

patient's history can be taken into account, and treatment decisions should always be based on individual considerations.

In our study, we show that combined CE-MR/CT is a cost-effective strategy for the staging of pancreatic cancer as compared to SCI using CE-CT. This finding was robust even considering realistic variations in induced costs as well as different probabilities of state transition. 


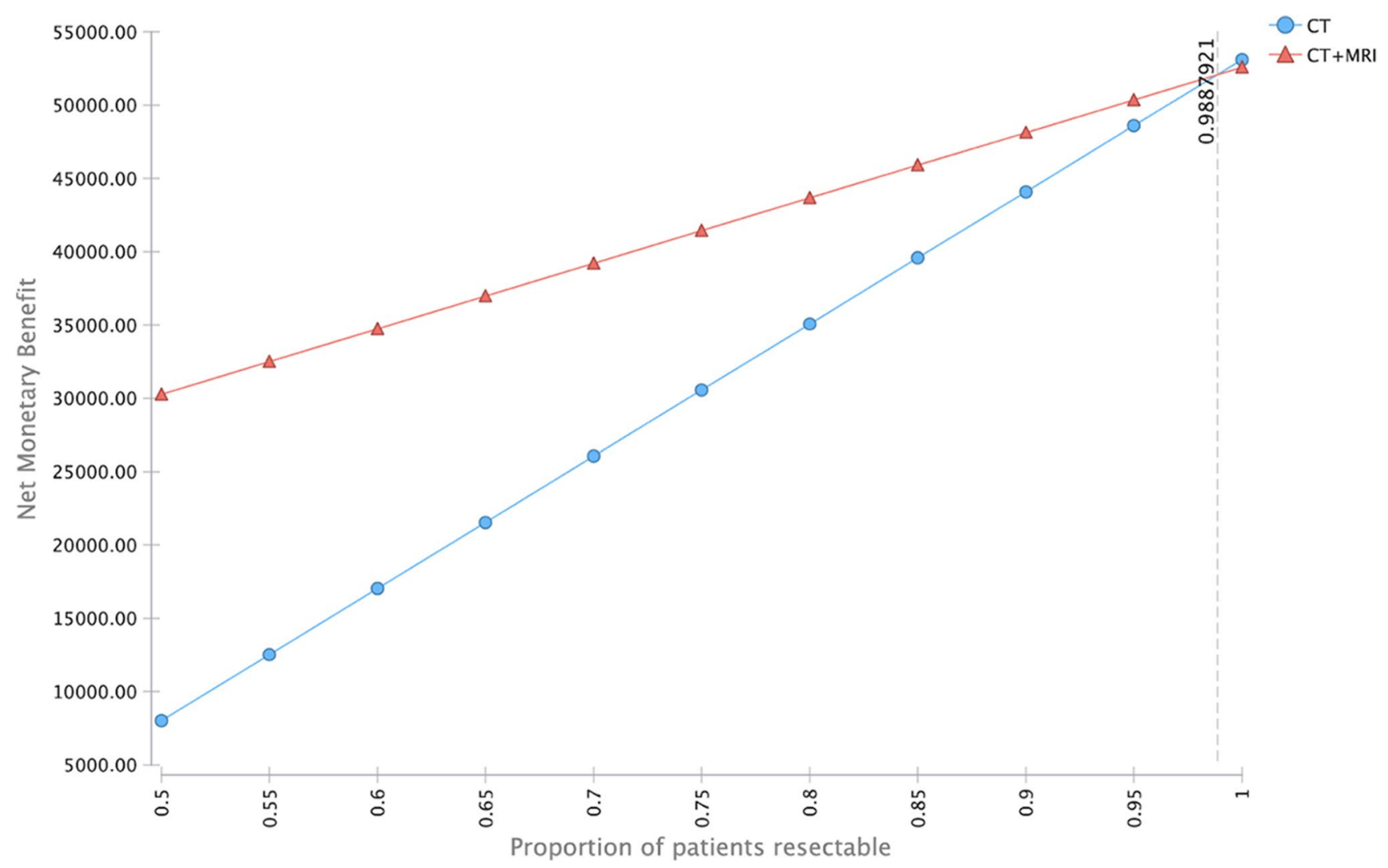

Fig. 4 Sensitivity analysis of the net monetary benefit (NMB) with respect to the probability of possible tumor resection. CE-MR/CT has a higher NMB up to a hypothetical resectability rate of higher than 0.98

Funding Open Access funding enabled and organized by Projekt DEAL.

\section{Declarations}

Guarantor The scientific guarantor of this publication is PD Dr. med. Matthias Frölich.

Conflict of interest The authors of this manuscript declare no relationships with any companies whose products or services may be related to the subject matter of the article.

Statistics and biometry Matthias Frölich has significant statistical expertise regarding cost-effectiveness analysis.

Informed consent Written informed consent was not required for this study due to retrospective analysis of commonly available data.

Ethical approval Institutional Review Board approval was not required due to retrospective analysis of commonly available data.

\section{Methodology}

- retrospective

- observational

- multicenter study
Open Access This article is licensed under a Creative Commons Attribution 4.0 International License, which permits use, sharing, adaptation, distribution and reproduction in any medium or format, as long as you give appropriate credit to the original author(s) and the source, provide a link to the Creative Commons licence, and indicate if changes were made. The images or other third party material in this article are included in the article's Creative Commons licence, unless indicated otherwise in a credit line to the material. If material is not included in the article's Creative Commons licence and your intended use is not permitted by statutory regulation or exceeds the permitted use, you will need to obtain permission directly from the copyright holder. To view a copy of this licence, visit http://creativecommons.org/licenses/by/4.0/.

\section{References}

1. Rawla P, Sunkara T, Gaduputi V (2019) Epidemiology of pancreatic cancer: global trends, etiology and risk factors. World J Oncol 10:10-27. https://doi.org/10.14740/wjon1166

2. Howlader N, Noone AM, Krapcho M et al (2020) SEER Cancer Statistics Review, 1975-2018, National Cancer Institute. Bethesda, MD, https://seer.cancer.gov/csr/1975_2018/, based on November 2020 SEER data submission

3. da Costa WL, Oluyomi AO, Thrift AP (2020) Trends in the incidence of pancreatic adenocarcinoma in all 50 United States Examined Through An Age-Period-Cohort Analysis. JNCI Cancer Spectr 4:1-7. https://doi.org/10.1093/jncics/pkaa033 
4. Pugalenthi A, Protic M, Gonen M et al (2016) Postoperative complications and overall survival after pancreaticoduodenectomy for pancreatic ductal adenocarcinoma. J Surg Oncol 113:188-193. https://doi.org/10.1002/jso.24125

5. Glasgow RE, Jackson HH, Neumayer L et al (2007) Pancreatic resection in veterans affairs and selected university medical centers: results of the patient safety in surgery study. J Am Coll Surg 204:1252-1260. https://doi.org/10.1016/j.jamcollsurg.2007.03. 015

6. Conroy T, Hammel P, Hebbar M et al (2018) FOLFIRINOX or gemcitabine as adjuvant therapy for pancreatic cancer. N Engl J Med 379:2395-2406. https://doi.org/10.1056/NEJMoa1809 775

7. Conroy T, Desseigne F, Ychou M, et al (2011) FOLFIRINOX versus gemcitabine for metastatic pancreatic cancer. N Engl J Med 1817-1825. https://doi.org/10.1056/NEJMoa1011923

8. Hess KR, Varadhachary GR, Taylor SH et al (2006) Metastatic patterns in adenocarcinoma. Cancer 106:1624-1633. https://doi. org/10.1002/cncr.21778

9. Zhang YJ, Huang J, Chen MS, Jiao LR (2012) Preoperative vascular evaluation with computed tomography and magnetic resonance imaging for pancreatic cancer: a meta-analysis. Pancreatology 12:227-233. https://doi.org/10.1016/j.pan.2012.03.057

10. Motosugi U, Ichikawa T, Morisaka H et al (2011) Detection of pancreatic carcinoma and liver metastases with gadoxetic acidenhanced MR imaging: comparison with contrast-enhanced multidetector row CT. Radiology 260:446-453. https://doi.org/10.1148/ radiol.11103548

11. Mao Y, Chen B, Wang $\mathrm{H}$ et al (2020) Diagnostic performance of magnetic resonance imaging for colorectal liver metastasis: a systematic review and meta-analysis. Sci Rep 10:1-12. https://doi. org/10.1038/s41598-020-58855-1

12. Kim YK, Park G, Kim CS, Yu HC, Han MY (2012) Diagnostic efficacy of gadoxetic acid-enhanced MRI for the detection and characterisation of liver metastases: comparison with multidetector-row CT. Br J Radiol 85:539-547. https://doi.org/10.1259/ bjr/25139667

13. Kim HJ, Park MS, Lee JY et al (2019) Incremental role of pancreatic magnetic resonance imaging after staging computed tomography to evaluate patients with pancreatic ductal adenocarcinoma. Cancer Res Treat 51:24-33. https://doi.org/10.4143/crt.2017.404

14. Arias E, Xu J (2020) United States Life Tables, 2018. Natl Vital Stat Rep 69:1-45

15. Kachare SD, Liner KR, Vohra NA, Zervos EE, Hickey T, Fitzgerald TL (2015) Assessment of health care cost for complex surgical patients: review of cost, re-imbursement and revenue involved in pancreatic surgery at a high-volume academic medical centre. HPB (Oxford) 17:311-317. https://doi.org/10.1111/hpb.12349

16. Sutton JM, Wilson GC, Paquette IM et al (2014) Cost effectiveness after a pancreaticoduodenectomy: bolstering the volume argument. HPB (Oxford) 16:1056-1061. https://doi.org/10.1111/ hpb.12309

17. Tramontano AC, Chen Y, Watson TR et al (2019) Pancreatic cancer treatment costs, including patient liability, by phase of care and treatment modality, 2000-2013. Medicine (Baltimore) 98(49):e18082. https://doi.org/10.1097/MD.0000000000018082

18. Howlader N, Noone A, Krapcho M, et al SEER Cancer Statistics Review, 1975-2017, National Cancer Institute. Bethesda, MD, https://seer.cancer.gov/csr/1975_2017/, based on November 2019 SEER data submission, posted to the SEER web site, April 2020

19. Suh $\mathrm{CH}, \mathrm{Kim}, \mathrm{KW}$, Park SH, et al (2018) Performing gadoxetic acid-enhanced MRI after CT for guiding curative treatment of early-stage hepatocellular carcinoma: a cost-effectiveness analysis. AJR Am J Roentgenol 210(2):W63-W69
20. O'Neill CB, Atoria CL, O'Reilly EM, LaFemina J, Henman MC, Elkin EB (2012) Costs and trends in pancreatic cancer treatment. Cancer 118:5132-5139. https://doi.org/10.1002/cncr.27490

21. Pezzilli R, Falconi M, Zerbi A et al (2011) Clinical and patientreported outcomes after pancreatoduodenectomy for different diseases: a follow-up study. Pancreas 40:938-945. https://doi.org/10. 1097/MPA.0b013e318216f693

22. Huang J, Yeo C, Sohn T et al (2000) Quality of life and outcomes after pancreaticoduodenectomy. Ann Surg 231:890-898

23. Carrato A, Falcone A, Ducreux M et al (2015) A systematic review of the burden of pancreatic cancer in Europe: real-world impact on survival, quality of life and costs. J Gastrointest Cancer 46:201-211. https://doi.org/10.1007/s12029-015-9724-1

24. Strobel O, Hank T, Hinz U et al (2017) Pancreatic cancer surgery. Ann Surg 265:565-573. https://doi.org/10.1097/SLA.0000000000 001731

25. Kovač JD, Mayer P, Hackert T, Klauss M (2019) The time to and type of pancreatic cancer recurrence after surgical resection: is prediction possible? Acad Radiol 26:775-781. https://doi.org/10. 1016/j.acra.2018.07.025

26. Sanders GD, Neumann PJ, Basu A et al (2016) Recommendations for conduct, methodological practices, and reporting of cost-effectiveness analyses: second panel on cost-effectiveness in health and medicine. JAMA 316:1093-1103. https://doi.org/10.1001/jama. 2016.12195

27. Cameron D, Ubels J, Norström F (2018) On what basis are medical cost-effectiveness thresholds set? Clashing opinions and an absence of data: a systematic review. Glob Health Action 11(1):1447828. https://doi.org/10.1080/16549716.2018.14478 28

28. Toft J, Hadden WJ, Laurence JM et al (2017) Imaging modalities in the diagnosis of pancreatic adenocarcinoma: a systematic review and meta-analysis of sensitivity, specificity and diagnostic accuracy. Eur J Radiol 92:17-23. https://doi.org/10.1016/j.ejrad. 2017.04.009

29. Bipat S, Saffire SKS, Van Delden OM et al (2005) Ultrasonography, computed tomography and magnetic resonance imaging for diagnosis and determining resectability of pancreatic adenocarcinoma: a meta-analysis. J Comput Assist Tomogr 29:438-445. https://doi.org/10.1097/01.rct.0000164513.23407. b3

30. McMahon PM, Halpern EF, Fernandez-del Castillo C, Clark JW, Gazelle GS (2001) Pancreatic cancer: cost-effectiveness of imaging technologies for assessing resectability. Radiology 221:93106. https://doi.org/10.1148/radiol.2211001656

31. Parikh T, Drew SJ, Lee VS et al (2008) Focal liver lesion detection and characterization with diffusion-weighted MR imaging: comparison with standard breath-hold T2-weighted imaging. Radiology 246:812-822. https://doi.org/10.1148/radiol.24630 70432

32. Muhi A, Ichikawa T, Motosugi U et al (2011) Diagnosis of colorectal hepatic metastases: comparison of contrast-enhanced CT, contrast-enhanced US, superparamagnetic iron oxide-enhanced MRI, and gadoxetic acid-enhanced MRI. J Magn Reson Imaging 34:326-335. https://doi.org/10.1002/jmri.22613

33. Heinrich S, Goerres GW, Schäfer M et al (2005) Positron emission tomography/computed tomography influences on the management of resectable pancreatic cancer and its cost-effectiveness. Ann Surg 242:235-243. https://doi.org/10.1097/01.sla.0000172095. 97787.84

34. Saing S, Haywood P, Duncan JK, Ma N, Cameron AL, Goodall S (2018) Cost-effective imaging for resectability of liver lesions in colorectal cancer: an economic decision model. ANZ J Surg 88:E507-E511. https://doi.org/10.1111/ans.14194 
35. Shiroiw T, Sung Y-K, Fukuda T, Lang H-C, Bae S-C, Tsutani K (2010) International survey on willingness-to-pay (WTP) for one additional QALY gained: what is the threshold of cost effectiveness? Health Econ. https://doi.org/10.1002/hec.1481

36. Gassert FG, Rübenthaler J, Cyran CC et al (2021) 18F FDG PET/MRI with hepatocyte-specific contrast agent for M staging of rectal cancer: a primary economic evaluation. Eur J Nucl Med Mol Imaging 48(10):3268-3276. https://doi.org/10.1007/ s00259-021-05193-7
37. Schnitzer ML, Froelich MF, Gassert FG et al (2020) Contrastenhanced CT after ablation of liver metastases of colorectal carcinoma - a cost-effectiveness analysis. Cancers (Basel). https:// doi.org/10.3390/cancers12092432

Publisher's note Springer Nature remains neutral with regard to jurisdictional claims in published maps and institutional affiliations. 